\title{
La gestión de placeres y daños: Estudio etnográfico sobre el consumo de drogas en vía pública, viviendas y salas de consumo higiénico
}

\author{
Managing pleasures and harms: An ethnographic study \\ of drug consumption in public spaces, homes and drug \\ consumption rooms
}

\section{Rafael Clua-García ${ }^{1}$}

'Doctor en Antropología social y cultural. Profesor asociado, Facultad de Ciencias de la Salud de Manresa, Universitat de Vic - Universitat Central de Catalunya, Manresa, España. $\triangle$ (iD)
RESUMEN Las salas de consumo higiénico $(\mathrm{SCH})$ son espacios para consumir drogas en condiciones higiénicas y seguras. Sin embargo, los usuarios alternan sus prácticas de consumo en vía pública y viviendas, entornos que conllevan mayores riesgos a la salud. Para comprender mejor este problema, se realizó un estudio etnográfico en diferentes espacios de consumo de Barcelona, incluyendo $\mathrm{SCH}$, vía pública y viviendas. Centrándonos en los discursos y prácticas de consumo, se recogieron datos mediante la observación participante y entrevistas semiestructuradas a 16 usuarios de las SCH. Los resultados muestran que, en los diferentes espacios de consumo, los usuarios experimentan varios tipos de placer. Además, estos espacios están asociados a diversos daños, los cuales son gestionados por los usuarios autorregulando sus prácticas. Estos aspectos, por lo tanto, deben tenerse en cuenta para diseñar acciones de reducción de daños adaptadas a las necesidades de los usuarios.

PALABRAS CLAVES Consumidores de Drogas; Reducción del Daño; Programas de Intercambio de Agujas; Investigación Cualitativa; Dependencia de Heroína; Cocaína; España.

\begin{abstract}
Drug consumption rooms (DCR) aim to facilitate consumption in hygienic and safe conditions. However, users also consume drugs in public spaces and homes generating incremental risk for health. To strengthen our understanding of consumption practices, we conducted an ethnographic study in different consumption locations in Barcelona, including DCRs, public spaces, and homes. Focusing on consumption practices and narratives, we conducted participant observation and interviewed 16 DCR users. Our findings show that different consumption spaces allow users to experiment different types of pleasures. In addition, consumption in each type of location is associated with various types of harms, which are managed by users by self-regulating their practices. These aspects, therefore, must be taken into account to design harm reduction action aligned with users' practices.
\end{abstract}

KEY WORDS Drug Users; Harm Reduction; Needle-Exchange Programs; Qualitative Research; Heroin Dependence; Cocaine; Spain. 


\section{INTRODUCCIÓN}

Desde principios de la década de 2000, los departamentos de atención a las drogodependencias de las agencias de salud pública de Barcelona y de Cataluña han coordinado, planificado y planteado directrices para fortalecer la red de programas de reducción de daños, dirigida a personas que usan drogas por vía parenteral y pulmonar ${ }^{(1,2)}$. Concretamente, se han incrementado los programas de intercambio de jeringuillas, como espacios para abastecer jeringuillas y otros utensilios para el consumo higiénico por vía parenteral y pulmonar y aproximar a los usuarios a servicios sociosanitarios ${ }^{(3)}$, y los programas de mantenimiento con metadona, para disminuir el consumo de opiáceos/opioides ilegales, reducir la incidencia de infección por $\mathrm{VIH}$ y hepatitis $\mathrm{B}$ y $\mathrm{C}$, reducir las muertes por sobredosis, disminuir las actividades delictivas y mejorar la calidad de vida de los usuarios $^{(4)}$. Ambos programas se han diversificado, sobre todo en el área metropolitana de Barcelona, en farmacias, unidades móviles y centros de atención y seguimiento a las drogodependencias, para mejorar el acceso y la cobertura de las necesidades de los usuarios de drogas ${ }^{(1)}$.

Además, como parte de la política de reducción de daños, se han puesto en marcha salas de consumo higiénico $(\mathrm{SCH})$ para consumir sustancias sometidas a fiscalización (heroína y cocaína), obtenidas en el mercado ilegal, en las que se garantiza un lugar y el material para el consumo higiénico, la supervisión y asistencia de profesionales en caso de sobredosis u otras complicaciones, y la promoción de hábitos saludables para reducir la morbimortalidad del consumo de dro$\operatorname{gas}^{(2,5,6,7,8,9)}$. Las $\mathrm{SCH}$ brindan un acercamiento de los usuarios a servicios de atención social y de salud, y disponen de zonas de "Calor y café" para atender sus necesidades básicas de higiene, alimentación y descanso ${ }^{(2,5,8)}$. Además, son dispositivos que mejoran el orden público, reduciendo la conflictividad comunitaria, las infracciones del consumo en vía pública y el número de jeringuillas y otras parafernalias abandonadas en lugares públicos $^{(5,8,10,11)}$.

La primera $\mathrm{SCH}$ en Cataluña se puso en marcha, en 2001, en una "escena abierta" de consumo de drogas situada en un barrio marginal de Barcelona, donde se concentraba la mayor parte de los consumidores de la ciudad $^{(12)}$. El derribo de este barrio, en 2004, provocó la dispersión de los consumidores por toda la ciudad, efecto que condujo a los técnicos municipales responsables a abrir nuevas $\mathrm{SCH}$ en diferentes distritos de la ciudad, así como a integrarlas en centros de atención de drogodependencias que ya existían, situados en las proximidades de zonas de venta y consumo de drogas ${ }^{(1,8)}$. En la actualidad, existen $13 \mathrm{SCH}$ en el territorio catalán, de las cuales 11 se integran en centros de reducción de daños y dos en unidades móviles y, en relación con la vía de consumo, 11 son para consumir por vía parenteral y dos por vía pulmonar ${ }^{(8,13)}$. En el último informe del sistema de información sobre drogodependencias de Cataluña de 2017, se registró un total de 3.176 usuarios diferentes que utilizaron las $\mathrm{SCH}$ y un total de 124.711 consumos, de los cuales 112.777 se realizaron por vía parenteral, 8.466 por vía pulmonar y 3.468 por vía intranasal, sin registrarse muertes por sobredosis ${ }^{(13)}$.

Los usuarios de las $\mathrm{SCH}$ son hombres en más de un $80 \%$, de edades comprendidas, mayoritariamente, entre los 30 y los 40 años, inmigrantes en más del $40 \%$, que presentan una prevalencia de más del $30 \%$ por $\mathrm{VIH}$, del $70 \%$ por VHC y alrededor del $30 \%$ coinfectados por $\mathrm{VIH}$ y $\mathrm{VHC}$. En general, se trata de una población con bajo nivel de instrucción, baja vinculación al mundo laboral, y que realizan actividades no legales como fuente de ingresos, que conllevan a problemas con la justicia $^{(2,8,14,15)}$. En estudios recientes se destacan diferencias entre los usuarios, según la intensidad de consumo, las condiciones sociosanitarias y la adherencia a las $\mathrm{SCH}$. En un estudio sobre el impacto de la frecuencia de asistencia a las $\mathrm{SCH}$ en relación con el consumo en vía pública, el riesgo de infección, sufrir sobredosis y acceder a servicios de las drogodependencias ${ }^{(2)}$, se detectó que más del $20 \%$ de los usuarios eran "frecuentes" y tenían 
un $61 \%$ menos de posibilidades de consumo en la vía pública con el riesgo que podría conllevar. En consecuencia, presentaban seis veces más posibilidades de desechar jeringas usadas en lugares seguros y tenían el doble de probabilidad de acceder a servicios de drogodependencias que asistentes "medios" y "bajos". Además, la proporción de participantes sin hogar era mayor en usuarios "frecuentes" que en los de frecuencia "media" y "baja", por lo que al no tener un lugar seguro para consumir podrían estar más dispuestos a usar regularmente las $\mathrm{SCH}$. En un estudio etnográfico se identificaron diferentes estilos de vida entre los usuarios de las $\mathrm{SCH}$ de Barcelona ${ }^{(14)}$. Entre los habituales a las $\mathrm{SCH}$, se detectaban usuarios con un patrón intenso que también consumían en vía pública y viviendas, que presentaban un deterioro físico destacable y no realizaban seguimientos de drogodependencias. Otros usuarios mantenían un consumo menos intenso, con fuerte adherencia a las $\mathrm{SCH}$, y realizaban seguimientos sociosanitarios. Entre los consumidores que usaban ocasionalmente las $\mathrm{SCH}$, se distinguían los que acudían para consumir en su trayecto diario y con baja adherencia al seguimiento de drogodependencias, caracterizados por no centrar su cotidianidad en el consumo y dedicarse a la familia, al trabajo u otras actividades, y otro grupo de usuarios con fuerte deterioro físico, en tratamiento de drogodependencias, que acudía en días indicados para consumir en las $\mathrm{SCH}$.

De este modo, a pesar de que las evidencias muestran que las $\mathrm{SCH}$ pueden contribuir a mejorar la salud y las condiciones sociales de los usuarios, se continúa consumiendo en espacios clandestinos e insalubres en vía pública y viviendas que son importantes en la ritualización del uso de sustancias, donde se experimentan altos riesgos y daños sociales y de salud. Este hecho destaca la necesidad de comprender, desde la perspectiva de los usuarios, las experiencias y prácticas de consumo, así como las estrategias de autoatención ${ }^{(16)}$ que se emplean en los diferentes espacios de consumo. Profundizar en estos aspectos permite entender los motivos y las experiencias de consumir en los diferentes contextos, así como en los autocuidados de los usuarios en las diferentes situaciones, aportándonos elementos para el diseño de estrategias de reducción de daños más efectivas. Para ello, en este artículo se tomó la perspectiva de los "entornos de riesgo" de Rhodes ${ }^{(17,18)}$, la cual considera que los daños del consumo se producen por factores sociales y estructurales, pero también políticos y económicos, que generan desigualdades y vulnerabilidad en las interacciones entre los usuarios de drogas y en los contextos de consumo. Bajo este enfoque, además de priorizar los determinantes sociales en la salud, se pone atención en la agencia para la prevención y las prácticas de consumo entre los usuarios de drogas en los diferentes entornos de riesgo ${ }^{(17,18,19)}$.

Esta perspectiva nos permite entender la gestión del riesgo como la capacidad de objetivar los eventos con el fin de hacerlos gobernables en los múltiples contextos y obtener experiencias positivas para el propio bienestar ${ }^{(19,20)}$. A través de la agencia, los usuarios de drogas participan de los diversos contextos de consumo, mediante la toma de decisiones según las condiciones materiales, sociales y culturales de las que disponen, y que definen los diferentes estilos de vida ${ }^{(14)}$. De forma variable, adquieren habilidades y prácticas para experimentar placeres y protegerse de los posibles daños del consumo de drogas en situaciones determinadas ${ }^{(19,21,22)}$. Concretamente, Ilevan a cabo acciones en las que se pone en juego el riesgo, como un proceso de mediación en las decisiones que pueden tener consecuencias positivas, en forma de placeres, o negativas, en forma de daños ${ }^{(20,23)}$. Específicamente, los placeres son el resultado de la intersección óptima entre los efectos esperados de las sustancias, de lo fisiológico, las formas de relacionarse, y la atracción y el compromiso con el contexto de consumo ${ }^{(22,24)}$. Los daños se consensuan entre los usuarios como las consecuencias negativas hacia la salud (infecciones de transmisión sanguínea, sobredosis, síndromes de abstinencia, etc.), así como los procesos de discriminación y estigmatización generados 
en el marco del prohibicionismo ${ }^{(25)}$. En dicha ecuación, el peligro infiere como un efecto indeseado no controlable por los usuarios de drogas, que puede ser manejado tomando acciones de reducción de daños ${ }^{(20,23)}$.

Este artículo es parte de un proyecto mayor titulado "¡Apúntame a la sala!: Epidemiología sociocultural del consumo de drogas y evaluación de las políticas de reducción de daños en las salas de consumo higiénico de Barcelona"(26). Derivados de esta investigación se han realizado dos trabajos previos al presente, uno sobre los "Estilos de vida de los usuarios de las salas de consumo higiénico de Barcelona"(14) y otro sobre la "Atención de enfermería del síndrome posconsumo de cocaína en usuarios de las salas de consumo higiénico de Barcelona"(27). El presente artículo se realizó con la misma muestra de participantes que el proyecto mayor; no obstante, la pregunta, los objetivos, la hipótesis y la metodología son diferentes a los trabajos ya publicados.

El objetivo de este artículo es analizar y comparar las prácticas de consumo y los discursos entre los usuarios de drogas que consumen en viviendas, vía pública y $\mathrm{SCH}$. Para ello, se profundiza a nivel micro en las dinámicas que rigen el consumo en los diferentes contextos, con el fin de esclarecer aspectos claves para modificar las condiciones sociales y promover intervenciones comunitarias que reduzcan el sufrimiento y la vulnerabilidad entre los usuarios de drogas en proceso de exclusión social. De este modo, se quiso comprender mejor los placeres que atraen a los usuarios a consumir en un determinado lugar y los daños que sufren en las diferentes situaciones, así como las estrategias de autoatención que emplean para autorregular los riesgos en cada caso. Este análisis aporta un conocimiento relevante para diseñar políticas de reducción de daños más adaptadas a las necesidades de los usuarios.

\section{METODOLOGÍA}

Se realizó un estudio cualitativo con enfoque etnográfico, método que permite un acerca- miento a la realidad y una interacción directa con los usuarios de drogas dentro de su cotidianidad ${ }^{(28,29,30,31)}$. Este enfoque hizo posible profundizar en los discursos y prácticas de consumo de los usuarios de drogas, permitiendo contrastar información y observaciones sobre los placeres y daños que experimentan y los métodos de reducción de daños que integran en los diferentes espacios de consumo. La investigación se realizó entre 2012 y 2016 en cinco centros de atención a las drogodependencias con SCH de Barcelona (Tabla 1) y sus alrededores (vía pública), situadas en tres zonas, con una fuerte escena de venta de drogas por las que circulan el mayor número de consumidores de drogas de esta área metropolitana.

La captación de los participantes del estudio se realizó durante la fase de familiarización de los diferentes ambientes de observación. Se llevó a cabo un muestreo cualitativo y teórico ${ }^{(33)}$. Durante el trabajo de campo se contactó a más de un centenar de consumidores de drogas por vía parenteral y pulmonar, de manera directa y por medio de la técnica "bola de nieve"(34), a través de usuarios y profesionales, y se entrevistaron de manera formal 16 usuarios de drogas. Participaron 9 hombres, cinco mujeres y dos transexuales con edades comprendidas entre 30 y 50 años, de los cuales seis eran consumidores de heroína, tres de cocaína y siete de ambas, y 12 eran consumidores por vía parenteral, tres por vía parenteral y pulmonar, y uno por vía pulmonar. En relación con las infecciones de transmisión sanguínea, ocho estaban coinfectados por $\mathrm{VIH}$ y hepatitis, tres infectados por $\mathrm{VHC}$, uno por $\mathrm{VIH}$ y cuatro referían no estar infectados. De los 16 participantes, 11 estaban en tratamiento de sustitución de opiáceos.

Se recogieron datos mediante la observación participante y entrevistas semiestructuradas. La observación participante implica observar y participar estableciendo una relación de compromiso/separación que se construye siguiendo las lógicas del trabajo de campo ${ }^{(35)}$. Para ello, se interaccionó con los usuarios de las $\mathrm{SCH}$ en las diferentes áreas de los dispositivos. Por ejemplo, se realizaron 
Tabla 1. Características de las salas de consumo higiénico estudiadas. Barcelona, 2012-2016.

\begin{tabular}{|c|c|c|c|c|c|}
\hline Características & SAPS & CAS Baluard & $\begin{array}{l}\text { Unidad Móvil de Zona } \\
\text { Franca }\end{array}$ & CAS Fòrum & $\begin{array}{l}\text { Centro Sociosanitario } \\
\text { "El Local" }\end{array}$ \\
\hline Ubicación & $\begin{array}{l}\text { Centro de la Ciudad de } \\
\text { Barcelona }\end{array}$ & $\begin{array}{l}\text { Centro de la Ciudad de } \\
\text { Barcelona }\end{array}$ & $\begin{array}{l}\text { Barrio periférico de } \\
\text { Barcelona }\end{array}$ & $\begin{array}{l}\text { Barrio periférico de } \\
\text { Barcelona }\end{array}$ & $\begin{array}{l}\text { Extrarradio, barrio de } \\
\text { La Mina de Sant Adrià } \\
\text { de Besòs }\end{array}$ \\
\hline Organización & Creu Roja & $\begin{array}{l}\text { Agència de Salut } \\
\text { Pública de Barcelona, } \\
\text { Associació Benestar i } \\
\text { Desenvolupament }\end{array}$ & $\begin{array}{l}\text { Agència de Salut } \\
\text { Pública de Barcelona, } \\
\text { Associació Benestar i } \\
\text { Desenvolupament }\end{array}$ & Parc Sanitari Mar & $\begin{array}{l}\text { Ajuntament de Sant } \\
\text { Adrià de Besòs, Institut } \\
\text { per a la Promoció Social i } \\
\text { de la Salut }\end{array}$ \\
\hline Horas de apertura (2015) & $\begin{array}{l}\text { De lunes a jueves de } \\
\text { 18:00 a 1:00, viernes de } \\
\text { 13:00 a 20:00 }\end{array}$ & $\begin{array}{l}\text { De lunes a viernes de } \\
\text { 7:00 a 22:00, fines de } \\
\text { semana de 8:00 a 19:00 }\end{array}$ & $\begin{array}{l}\text { De lunes a viernes de } \\
14: 00 \text { a 21:30 }\end{array}$ & $\begin{array}{l}\text { De lunes a domingo de } \\
12: 15 \text { a } 18: 45\end{array}$ & $\begin{array}{l}\text { De lunes a viernes de } \\
\text { 11:00 a 19:00, fines de } \\
\text { semana de 11:00 a 15:00 }\end{array}$ \\
\hline Plazas de la SCH (2015) & $\begin{array}{l}2 \text { (consumo por vía } \\
\text { parenteral) }\end{array}$ & $\begin{array}{l}5 \text { (consumo por vía } \\
\text { parenteral), } \\
6 \text { (consumo por vía } \\
\text { pulmonar) }\end{array}$ & $\begin{array}{l}3 \text { (consumo por vía } \\
\text { parenteral) }\end{array}$ & $\begin{array}{l}1 \text { (consumo por vía } \\
\text { parenteral) }\end{array}$ & $\begin{array}{l}8 \text { (consumo por vía } \\
\text { parenteral) }\end{array}$ \\
\hline $\begin{array}{l}\text { Programas y áreas del } \\
\text { dispositivo }\end{array}$ & $\begin{array}{l}\text { PIJ, Enfermería y “Calor } \\
\text { y café" }\end{array}$ & $\begin{array}{l}\text { PIJ, PMM, Enfermería y } \\
\text { "Calor y café" }\end{array}$ & PIJ & $\begin{array}{l}\text { PIJ, PMM, Enfermería y } \\
\text { "Calor y café" }\end{array}$ & $\begin{array}{l}\text { PIJ, Enfermería y "Calor } \\
\text { y café" }\end{array}$ \\
\hline Número de usuarios (2015) & 415 & 1.875 & 94 & 625 & 1.967 \\
\hline $\begin{array}{l}\text { Número de consumos en SCH } \\
\text { (2015) }\end{array}$ & 1.650 & 19.031 & 1.155 & 4.052 & 64.553 \\
\hline
\end{tabular}

Fuente: Elaboración propia sobre la base de la bibliografía seleccionada $(8,32)$.

CAS = Centro de Atención y Seguimiento a las Drogodependencias; PIJ = Programa de Intercambio de Jeringuillas; PMM = Programa de Mantenimiento con Metadona; SAPS = Servei d'Atenció i Prevenció Socio Sanitària.

observaciones y se interaccionó con los usuarios en las consultas de enfermería, en los puntos de intercambio de jeringuillas y en los espacios de consumo; se asistió a talleres de prevención de sobredosis; se estableció conversación con los usuarios en el momento de realizar el consumo en las $\mathrm{SCH}$ o al intercambiar material de consumo en los programas de intercambio de jeringuillas y se tomó contacto con ellos en los "Calor y café" (zonas de descanso), programas de intercambio de jeringuillas o $\mathrm{SCH}$ para proponer la realización de entrevistas. Además, se realizaron observaciones en vía pública, próxima a las $\mathrm{SCH}$, para conocer las prácticas de consumo y discursos de los usuarios que en ocasiones no acuden a estos dispositivos. Para ello se acompañó a los educadores de calle que realizan intervención comunitaria y acuden a espacios de consumo para la recogida de jeringuillas usadas, realizándose observaciones de las prácticas del consumo en vía pública (portales, descampados, etc.) y entablando conversación con los usuarios de drogas. El registro de estas sesiones se plasmó en un diario de campo para describir los hechos, los acontecimientos, los lugares y personas, e interpretar las impresiones y las reflexiones del investigador ${ }^{(36)}$. En total se realizaron 259 sesiones, redactadas en 1.928 páginas del diario de campo.

Mediante las entrevistas semiestructuradas $^{(37,38)}$ se obtuvo información específica sobre las prácticas de consumo, las representaciones y los discursos relacionados, en un contexto relativamente íntimo que las personas que consumen drogas por vía parenteral y pulmonar no siempre están dispuestas a dar en forma colectiva, que permitió complementar los datos recogidos vía observación participante. Para ello se diseñó un guion a partir de los datos obtenidos mediante la observación participante, en el que se exploraron las narrativas y las representaciones relacionados con los placeres y los daños, y las estrategias de autoatención entre los participantes. En las entrevistas se operacionalizó el placer como los beneficios 
del consumo de drogas, las relaciones que se establecen y las experiencias positivas en los diferentes contextos; el daño, como los peligros y los efectos negativos del consumo en determinadas situaciones; $y$ las estrategias de autoatención, como las formas de reducir los riesgos y daños.

Cada entrevista se dividió en tres bloques temáticos que incluía preguntas deductivas en torno al consumo de drogas en los diferentes espacios de consumo: 1) Consumo de drogas en viviendas, por ejemplo, “¿cuáles son los peligros de consumir en una casa?", "¿qué medidas tomas para prevenir una sobredosis?"; 2) Consumo de drogas en vía pública, por ejemplo, "¿qué ventajas tiene consumir en la calle?", "ante situaciones de peligro en la calle, ¿qué métodos tomas para protegerte?"; y 3) Consumo de drogas en $\mathrm{SCH}$, por ejemplo, "¿por qué tomas la decisión de acudir a una sala consumo?", "¿qué beneficios tiene consumir en una sala de consumo?". Las entrevistas fueron grabadas digitalmente y transcritas en forma textual para su posterior análisis. Se tomaron en cuenta consideraciones éticas con respecto a la confidencialidad y el anonimato de los participantes de la investigación, reemplazando los nombres reales por nombres ficticios.

Se realizó un análisis de contenido de los materiales recogidos. Este consistió en la interpretación y la comparación objetiva y sistemática de los datos en términos de algún denominador común ${ }^{(39)}$. Las notas de campo y las entrevistas semiestructuradas fueron codificadas elaborando un índice de conceptos con información de carácter provisional. Estos códigos se ordenaron y se compararon entre ellos para simplificarlos y obtener códigos más precisos. Estos permitieron identificar descripciones e ideas que se agruparon en familias temáticas, generando categorías que proporcionaron una explicación teórica de los datos con relación al objeto de estudio. La credibilidad y validez de los datos analizados fueron incrementadas mediante la triangulación ${ }^{(40)}$, articulando los datos recolectados mediante diferentes técnicas de obtención de datos en varios espacios de consumo y con múltiples usuarios de drogas.
Todos los entrevistados firmaron un consentimiento informado y un documento en el que se informaba el objetivo de la investigación y sobre el uso de los datos recogidos. Estos documentos fueron aprobados por la Comisión de Ética de la Universitat Autònoma de Barcelona.

\section{RESULTADOS}

De acuerdo con el enfoque propuesto y basado en los "entornos de riesgo", los resultados se fundamentan en la descripción de los diferentes contextos de consumo, descritos anteriormente. El análisis de los datos bajo este enfoque permite entrelazar tres dimensiones centrales de la relación entre consumo y espacio, y que estructuran el desarrollo de cada apartado: la primera dimensión se centra en los aspectos positivos y placenteros del consumo en un entorno concreto; una segunda, en la que se contraponen los aspectos negativos y los daños del consumo en ese espacio; $y$, una tercera, en la que se analizan las estrategias de autoatención que toman los usuarios para gestionar los riesgos en cada uno de los espacios de consumo.

\section{Consumo en vía pública}

El consumo de drogas en vía pública representa un tipo de práctica de alto riesgo social y para la salud. Se tratan de lugares con bajas condiciones higiénicas, que permiten a los usuarios ocultarse en portales de viviendas, edificios abandonados y zonas verdes en las proximidades de los puntos de venta de drogas. Estos espacios son escogidos por los usuarios para los que "consumir en la calle es sensacional" frente a aquellos que, por el contrario, consideran dicha práctica como "peligrosa", prefiriendo consumir en espacios considerados más seguros, como pueden ser las $\mathrm{SCH}$ o viviendas. Sin embargo, en ocasiones no existe esta posibilidad, dado que muchos usuarios no tienen vivienda o no pueden consumir en $\mathrm{SCH}$, dadas sus 
limitaciones horarias (Tabla 1), o por haber sido expulsados de estos dispositivos por incumplir gravemente las normas o haber provocado episodios de violencia hacia otros usuarios o profesionales. En estos casos, los usuarios toman medidas de seguridad para evitar daños del consumo en vía pública.

\section{"Consumir en la calle es sensacional"}

Los usuarios con un patrón intenso de consumo son los que habitualmente realizan sus prácticas en vía pública. En general, son usuarios de cocaína y heroína por vía parenteral que explican que consumir en la calle les permite una sensación más intensa en un entorno relativamente seguro. Estos acuden a lugares próximos a las casas de venta de drogas, como portales, parques u otros espacios donde guarecerse, para consumir de forma inmediata. Ritualizan la práctica del consumo libremente bajo las propias decisiones y las condiciones que permiten los ambientes escogidos. En estos, los usuarios acuden solos o acompañados de otros usuarios con los que comparten la compra de sustancias, o se unen con otros grupos que inspiran confianza. En relación con los consumidores de cocaína, argumentan que, en la calle, la experiencia es más tranquila y una forma de obtener efectos más intensos. En la calle se consume con mayor libertad y, en el caso de sufrir paranoias, refieren que el consumo es menos estresante que en un espacio cerrado por paredes. En relación con el consumo de heroína, argumentan que en la calle es posible experimentar efectos placenteros más intensos, evitando las impertinencias y ruidos de personas con las que no se ha decidido compartir el espacio.

Mira, ¿ves esto? (indicándome un bordillo situado entre unos matorrales) Aquí suelo venir yo. Aquí se está bien. Queda apartado y está más o menos limpio. Aquí estás a tu rollo, estás más tranquilo, con tu cuelgue (embriaguez) y nadie te da voces, ni ruidos... estás al aire libre sin que nadie te diga nada y disfrutando. (Nota de campo, conversando con José María en vía pública).
La ventaja es... es otra manera, porque ahí [en la $\mathrm{SCH}$ ] con los chillidos y los gritos no te lo pones a gusto. En la calle te pones en un rincón, estás pendiente de la gente, pero te lo pones más a gusto. (Julián)

En la calle es más tranquilo, puedes consumir donde quieras, te puedes esconder... Bueno, puedes estar en grupo y puedes hablar con más gente, estar 7 u 8 personas... estar hablando, estar a tus anchas... Aunque la sala esté abierta, por las prisas te vas... si acabas de pillar [comprar drogas], pues como estás al lado, consumes al lado... (Javier)

\section{"Consumir en la calle es peligroso"}

Los usuarios que describen el consumo en vía pública como "peligroso" son aquellos que tienen un patrón de consumo menos intenso que los que prefieren consumir en la calle. Respecto al consumo de cocaína por vía parenteral, refieren que cuando se sufren "paranoias" es difícil mantener la calma para soportar tales efectos en un ambiente desprotegido. Entre los consumidores de heroína por vía parenteral, se baraja el riesgo de sufrir una sobredosis fatal y tener dificultades de ser atendido para evitar la muerte. En ambos casos, refieren que las bajas condiciones de higiene en vía pública puede conllevar a re/infectarse de VIH y/o hepatitis B y C. Entre los consumidores de drogas por vía pulmonar, aunque no se contemplan estos riesgos, refieren que este tipo de consumo es difícil de realizar en condiciones meteorológicas adversas (viento, lluvia, etc.), por lo que muchos prefieren consumir en viviendas o $\mathrm{SCH}$.

El problema es que te puede ver la gente, a veces hay críos que te pueden ver. Aparte que la gente es muy guarra porque se dejan las jeringuillas tiradas por cualquier lado y te puedes pinchar con cualquier cosa. Tienes que ir con 40 mil ojos. Con la policía también tienes que ir esquivándolos. (Cris) 
En la calle no hay ninguna ventaja... tienes la desventaja que te interrumpan, sobre todo consumiendo caballo [heroína]... te interrumpen en tu ritual, no estás tranquilo, te estás quemando... ¡pum! la goma [torniquete], que no me vean, ipum! que viene alguien... recoge todo que no se caiga, que ipum! que ¡pam!, es horrible y la policía que te puede pillar. Además, si te da una sobredosis y si no lleva naloxona el colega o no hay nadie cerca o cualquier cosa y te quedas. Ese es el peligro. (Mick)

En la calle es muy difícil fumarse allí un chino [fumar heroína] porque tienes que buscarte un sitio de ladrillos para meterte ahí en medio. Es más difícil fumar porque se estropea, si no encuentras un sitio cerrado, pierdes mucho. En la calle no hay ninguna ventaja. Tampoco me gusta que te vean, que causa mala imagen. No, no le veo ninguna ventaja. (Kike)

Vinieron unos y yo estaba abriendo una bola [dosis] con una navaja. Uno me la pidió... el muy hijo de puta se me encaró y me dijo "dame la bola, pero ya" y yo le dije "tío, va, déjate de bromas" y otro me dijo "oye tío, que va en serio...". El hijoputa, me quitó la riñonera, la bola [dosis] porque me levantó del suelo y me empezó a zarandear. (Nota de campo, conversando con Juanito en el "Calor y café")

Los usuarios contrarios al consumo en la caIle remarcan que en estos espacios se corre el riesgo de ser sorprendidos por vecinos y transeúntes y a tener problemas de orden público al ser interceptados por la policía. Además, los usuarios que realizan consumos en solitario refieren el riesgo de sufrir agresiones o extorsiones por parte de otros usuarios.

\section{Estrategias de autoatención en vía pública}

Para evitar consecuencias sociales negativas, los usuarios suelen buscar un lugar tranquilo para consumir en compañía y alejados de miradas de vecinos y transeúntes. En el caso de ser sorprendidos por la policía, procuran dar explicaciones razonables de por qué consumen en vía pública. En el caso de ser registrados e interceptarles las dosis de droga, suelen alegar que es para consumo propio para evitar sanciones, y en caso de que los agentes presionen a que respondan dónde han adquirido las sustancias, utilizan información falsa como moneda de cambio para evitar incautaciones y delatar al vendedor de drogas.
Siempre busco un lugar tranquilo. Nor- malmente con alguien porque la cocaína te da por estar con alguien, si estás solo te rayas. Te gusta estar en compañía y hablar con la gente y estar a tu rollo, con tu paranoia y tu colocón. (Jonatán)

Yo me pincho en un parque que no pase nadie. El único peligro es la policía y si vienen les explico "oye mira, soy toxicómano y me estoy pinchando y no voy a tirar la jeringuilla y si me la quitas, voy a tener el mono [síndrome de abstinencia], y me voy a tener que ir a robar y vais a tener trabajo". A la policía si tú les hablas bien ellos se comportan. A lo mejor hay algunos que no, pero si tú les hablas claro, todo bien. (Miguel)

Ya voy con cuidado y tomo mis seguridades. Si quisiera caerme de boca ya lo haría, tampoco hago el doble tirabuzón... me pongo lo justo sin ponerme malo... Ya miro de hacer bien las cosas. Antes estaba liadísimo, robando y así todos los días, ahora lo justo para no liarla. (Nota de campo, Cristian conversa con un educador de calle en vía pública).

En estos espacios llevan a cabo una serie de autocuidados para reducir daños, ante la imposibilidad de acudir a espacios considerados más seguros. En relación con la prevención de las enfermedades infectocontagiosas, procuran ir provistos de material estéril para la preparación y consumo de drogas por vía parenteral. Sin embargo, no siempre sucede así, detectándose prácticas de riesgo como compartir jeringuillas y otras parafernalias 
para la preparación de la inyección entre los usuarios. Respecto a las sobredosis, gestionan el consumo de drogas evitando cantidades de sustancia que no puedan tolerar que produzcan sobredosis u otros efectos adversos. En pocas ocasiones refieren llevar kits de naloxona para actuar en caso de sobredosis de heroína, si bien, suelen llevar teléfonos móviles para llamar a servicios de urgencias o realizan el consumo en zonas próximas a paseos donde pueden alertar a transeúntes o a las $\mathrm{SCH}$ para avisar a los profesionales. Entre los consumidores por vía pulmonar, para vencer las condiciones meteorológicas adversas, realizan sus prácticas en lugares resguardados, como dentro de vehículos o marquesinas.

\section{Consumo en viviendas}

Este tipo de consumo es el que se realiza en casas particulares, casas okupas y pisos de venta de drogas. En cada uno de estos espacios existen matices en la organización del consumo. Las casas particulares son los espacios menos frecuentados por los usuarios dado que muy bajo porcentaje vive en este tipo de viviendas. En estos casos, se trata de usuarios que consumen de forma moderada, en solitario o en pequeños grupos, que consideran estos espacios seguros e íntimos. Las casas okupas son espacios frecuentados por grupos extensos de consumidores, más o menos organizados. Respecto a los pisos de venta, existen diferencias en el tipo de vivienda entre los diferentes barrios estudiados. En el centro de la ciudad de Barcelona, se detectan pisos en condiciones de infravivienda organizados exclusivamente para vender y consumir, donde se reúnen pequeños grupos de consumidores. En barrios periféricos o en el extrarradio de la ciudad, se detectan viviendas en las que se diferencian los espacios para la vida doméstica de los espacios para la venta de drogas. En este tipo de viviendas solo se permite el consumo a usuarios de confianza, sin concentración de grupos extensos. En general, los usuarios valoran que consumir en viviendas es más "tranquilo" que consumir en la calle o en
$\mathrm{SCH}$. Por el contrario, otros usuarios refieren haber experimentado situaciones "problemáticas", por lo que buscan espacios más seguros y, llegado el caso, toman medidas de reducción de daños.

\section{"Consumir en casa es tranquilo"}

El consumo de drogas en una vivienda permite la preparación y consumo de drogas con tranquilidad, sin ser molestado por otros usuarios y sin tener que asumir las normas de uso de las $\mathrm{SCH}$. En comparación al consumo en vía pública, los usuarios de drogas refieren que consumir en viviendas es una forma de evitar ser sorprendidos por los vecinos y la policía, sin interrupciones, sin recibir recriminaciones y de poder experimentar efectos deseados sin contratiempos. En general, los usuarios que consumen en viviendas son aquellos que realizan un consumo intenso de drogas, sobre todo aquellos que viven en casas okupas, o aquellos que a menudo consumen en vía pública pero que acuden a casas de venta de drogas, donde se permite consumir, situadas en barrios con fuerte control policial. En estos espacios es posible consumir de forma oculta y disfrutar los efectos de las sustancias en pequeños grupos.

Yo, donde pillo [comprar drogas] muchas
veces me dejan fumármelo [heroína por
vía pulmonar]. Así no tengo que hacerlo
en las escaleras de los bloques y que me
vean los niños o broncas con los gitanos
por estar andando por el portal. (Nota de
campo, conversando con José en el Pro-
grama de Intercambio de Jeringuillas).

La primera noche que llegué aquí, a Barcelona, ya me llevó un taxista a un piso donde se vende y se puede fumar $y$ se puede inyectar allí. Tienen cubos de esos amarillos para recoger chutas [jeringuiIlas]. Y siguen allí, lo conocí a los muy pocos días... yo voy, compro y fumo. (Kike)

Cuando vivía con mi novia, estábamos en una habitación y consumíamos allí los dos. Para mí, la ventaja es que no 
tienes la paranoia de la gente, de que la gente te observa, te mira, etc. Estás en tu casa y estás tranquilo y no tienes la paranoia esa, la obsesión. Yo, por ejemplo, cuando me meto pienso que me miran, se ríen de mí. La cocaína es paranoia total. (Jonatan)

En la ciudad de Barcelona solo existe una $\mathrm{SCH}$ para el consumo por vía pulmonar, la cual no está abierta 24 horas. De este modo, cuando están cerradas o los usuarios adquieren sustancias en barrios que no disponen de estos espacios, se deben buscar alternativas, como consumir en viviendas. Además, el consumo en estos espacios es una buena forma de evitar las condiciones meteorológicas adversas y las inconveniencias con la policía y los transeúntes.

\section{“Consumir en casa es problemático"}

El consumo de drogas en viviendas reporta consecuencias negativas sociales y para la salud. Los consumidores de heroína por vía parenteral refieren que en estos espacios se corre el riesgo de sufrir sobredosis, sin contar con medios suficientes para revertirlas, aún más si se consume solo o con personas con poca capacidad para la atención. En el caso de los consumidores de cocaína, tanto por vía pulmonar como por vía parenteral, refieren que se corre el riesgo de sufrir graves alucinaciones y ansiedad difíciles de ser aliviadas en este tipo de entorno.

Yo vengo aquí [ $\mathrm{SCH}$ para el consumo por vía pulmonar] porque vivía en una casa con varios pakistaníes y no se podía fumar... un día uno me vio lo que hacía y no le parecía bien y me dijo que no me quería en la casa. Al final me fui de esa casa y estuve una semana viviendo en la calle hasta que encontré una habitación. Pasé de pagar 250 euros a 300, por su culpa, pero ahora puedo fumar en mi habitación porque estoy solo en una para mí. (Nota de campo, conversando con Ahmed en la $\mathrm{SCH}$ ).
La familia sabe que me drogo, pero nunca me han visto, se lo huelen, pero pienso que nunca me han visto. He consumido otras veces en el comedor, pero porque estaba ya desesperado... normalmente, he preferido bajar y pincharme en la calle y subir a casa. (Miguel)

En una casa igual te encierras en la habitación y te encuentran ahí muerta, nada. Tiene que haber siempre una persona ahí al lado, pero una persona que sepa, no un toxicómano... un toxicómano te va a quitar lo que tengas si te da sobredosis y le tienes que invitar o algo porque un toxicómano no va a estar él al lado tuyo consumiendo y él mirándote. (Esmeralda)

Los usuarios relatan una serie de inconvenientes según el tipo de vivienda. En casas particulares compartidas con familiares o compañeros de piso que no son usuarios de drogas, refieren que ser sorprendido consumiendo puede conllevar problemas de convivencia. En las casas okupas, suceden situaciones similares, sobre todo cuando no se respetan los espacios comunes o se han realizado consumos preservando pocas medidas de seguridad e higiene. En los pisos de venta, lugares donde las estancias no son prolongadas, el mayor problema es cuando se producen altercados entre consumidores y vendedores o cuando se realizan redadas policiales con múltiples detecciones.

\section{Estrategias de autoatención en viviendas}

En los casos que se decide consumir en una casa, aún sabiendo que se corre el riesgo de sufrir una sobredosis de heroína o una reacción adversa a la cocaína, los participantes manifiestan una serie de medidas de protección similares a las tomadas cuando se consume en la calle. En general, se procura adquirir material higiénico de consumo antes de ir a comprar o consumir drogas y se organiza el espacio con ciertas medidas de higiene (disponer de contendores para jeringuillas usadas). Para mayor seguridad, se 
consume acompañados con gente de confianza y llevan teléfonos móviles para poder comunicarse con un servicio de urgencias en caso de sobredosis. En aquellos que viven en pisos particulares y casas okupas, se procura consumir en horas que no haya presencia de familiares y compañeros de piso que puedan recriminar este tipo de prácticas y así evitar ser sorprendido y no generar malestar en la convivencia.

Yo ya no consumo como antes, me pego mi homenaje, pero no me gusta hacerlo aquí $[\mathrm{SCH}]$. A mí no me gustan las salas de consumo porque la peña te pide y te agobia. Yo lo hago en mi casa. Siempre me pongo el 112 [número de emergencias] en el móvil, por si acaso. Hombre, yo no me quiero morir. (Nota de campo, conversando con Manolito en vía pública)

Mi mujer cree que ya no consumo... sabe que he tomado drogas... Cuando estoy en casa en vez de hacerme un chino [fumar heroína], me voy al lavabo y me pongo una raya... ya hago para que no se entere. (Nota de campo, conversando con Said en la $\mathrm{SCH}$ )

Traigo dos cubos con chutas [contenedores con jeringuillas usadas]... necesitamos dos contenedores más y llevarnos chutas (jeringuillas), aguas [bidestilada]... Ahora estoy viviendo con unos amigos en una [casa] okupa, queremos que esté todo recogido. (Nota de campo, Marcelo conversando con una educadora social en el programa de intercambio de jeringuillas)

\section{Consumo en salas de consumo higiénico}

Las $\mathrm{SCH}$ son espacios para consumir de forma higiénica y segura bajo supervisión de profesionales. Los usuarios que acuden a las $\mathrm{SCH}$ refieren que "consumir en la sala de consumo es seguro", mientras que los que son contrarios a estos espacios, refieren que el consumo es "molesto". En estos casos, toman medidas para que acudir a las $\mathrm{SCH}$ sea favorable.

\section{"Consumir en la sala de consumo es seguro"}

Los usuarios de las $\mathrm{SCH}$ suelen ser personas que usan drogas por vía parenteral, que presentan deterioro físico y dificultades para realizar la práctica de consumo de forma satisfactoria en otros espacios. Estos refieren que en las $\mathrm{SCH}$ se reduce el riesgo de sobredosis y, en caso de sufrirlas, se dispone de profesionales que supervisan y asisten para evitar casos fatales. Allí disponen de material higiénico gratis y los profesionales ayudan a realizar la práctica del consumo con menos daños (búsqueda de zonas de punción, canalización de la vena, etc.). Entre los usuarios por vía pulmonar se afirma que en las $\mathrm{SCH}$ se puede consumir de manera satisfactoria y tranquila y disponer de material específico para esta práctica. En todos los casos, refieren que en las $\mathrm{SCH}$ se dispone de espacios para descansar tras el consumo y es posible pedir ayuda sanitaria, social y jurídica. Consideran que al consumir en un espacio tranquilo y seguro es posible disfrutar de los efectos deseados en condiciones óptimas.

Consumir en la calle es que lo veo un mal ejemplo, lo veo fatal porque lo ve un niño, lo ve cualquiera, te ve todo el mundo. Yo esto de la sala $[\mathrm{SCH}]$ lo veo muy bien. A mí dame sala. Además, es más higiénico, nadie te molesta y "yastá". No por ahí tirada en el suelo, hecho un engendro. (Vanesa)

Vengo a la sala $[\mathrm{SCH}]$ por la asistencia médica. Es una seguridad para los consumidores, es una forma de hacerlo bajo control, y una forma que dentro de lo sano que sea menos malo. Aquí $[\mathrm{SCH}]$ estás protegida por gente muy especial, ¿sabes? Que nos cuidan. Nos puede pasar cualquier cosa... (Berta) 
Viniendo aquí mejoras en salud, mejoras en dinero, y tranquilidad... dinero porque ya tienes ahí las insulinas, las chutas [jeringuillas], la goma, el agua, tienes para limpiarte... Estás protegido, la policía sabe que ahí se están drogando, pero no pueden entrar. Tienen un respeto al sitio. (Miguel)

Por seguridad, por tranquilidad... no por seguridad a nivel de sobredosis, seguridad a nivel policial. Si estás ahí, no debes tener miedo de que haya una redada que venga la policía, de que tal. Es un sitio donde fumas [consumo de drogas por vía pulmonar] más tranquilo. (Kike)

Al acudir a las $\mathrm{SCH}$, los usuarios evitan los riesgos sociales del consumo en viviendas o vía pública. Se evita tener problemas de convivencia con compañeros de piso o ser sorprendido por la policía, evitando multas u otros problemas de orden público.

\section{"Consumir en la sala de consumo es molesto"}

Los usuarios contrarios a las $\mathrm{SCH}$ señalan que acudir a estos espacios es molesto por diversos motivos. La principal limitación son las normas de las $\mathrm{SCH}$. Por ejemplo, en la mayoría de las $\mathrm{SCH}$ existe un límite de tiempo para preparar y consumir (30 minutos para el consumo por vía parenteral y 45 para el consumo por vía pulmonar) y una vez que se ha consumido no se permite volver a consumir hasta que no pase un intervalo de 30 minutos. Para muchos usuarios estas regulaciones desmotivan a consumir en las $\mathrm{SCH}$, prefiriendo hacerlo en otros lugares. Otros usuarios refieren que, a veces, hay problemas en la interacción con los profesionales, relacionado con la rigidez del cumplimiento de las normas o supervisión extrema, que impide disfrutar del consumo según sus expectativas. Algunos usuarios refieren que los profesionales están constantemente recriminando las prácticas y comportamientos experimentados bajo los efectos de las sustancias. Entre los consumidores de cocaína se extiende el argumento que, dentro de una $\mathrm{SCH}$, los efectos de esta sustancia se experimentan de forma negativa produciendo malestar a sí mismo y hacia el resto de los usuarios y profesionales.

Los profesionales están ahí y te ayudan, que si no, no vendría. Lo que pasa que cuando hay cola, te vas al monte... que si tienes que esperar, que uno prepare, que si el otro tal. No te puedes fiar, si vas a medias, el otro se carga la suya bien y te da la que no lleva nada. (Ramón)

Las narcosalas $[\mathrm{SCH}]$ que hay aquí en Barcelona, están hechas para gente que consume heroína. No puedes poner a alguien que consume cocaína con uno de heroína... al de la cocaína, le da un efecto diferente que al de la heroína. Con eso te relajas y la cocaína es paranoica y a cada uno le da por rascarse, por mirar, y tú te sientas y te pega y te empiezan "va, venga, levanta", "venga, recoge la mesa" y el otro te pide... eso hace que cuando te enchufas no estés a gusto. (Edgar)

La desventaja es la pesadez de la gente que no son tus amigos, ni nada, que están "eh Mick... dame un uno", "que no tío, ni uno ni dos" es un poco rollo depende de quién haya. Están los "helicópteros" que ya antes de llegar "oye tío, por favor, que no se qué, que pum", "oye mira..." y el problema es que lo has invitado algún día y luego tú le pides un poco y te dicen "no, no que solo tengo medio", "pero, si es que solo te pido un 0,2 [mililitros]...", es un rollo. (Mick)

Además, al acudir a una $\mathrm{SCH}$ se corre el riesgo de tener problemas con otros usuarios en diversas situaciones, como recibir acosos y peticiones de invitación, tener discrepancias en la repartición de dosis cuando se han comprado drogas a medias, así como estar en un ambiente con ruidos y altercados. Otro motivo para no acudir a las $\mathrm{SCH}$, son los controles policiales rutinarios en las proximidades de estos dispositivos. Los usuarios refieren que este tipo de prácticas suponen una 
barrera de acceso, por el miedo a ser identificados o detenidos en el caso de tener una petición de búsqueda y captura.

\section{Estrategias de autoatención en las salas de consumo higiénico}

Ante las diferentes situaciones "molestas" los usuarios toman una serie de medidas en caso de acudir a las $\mathrm{SCH}$. Para evitar aglomeraciones y tiempo de espera, los usuarios acuden a centros de reducción de daños con poca afluencia y con pocas plazas de consumo (Tabla 1), en los que es posible consumir más tranquilamente, sin aglomeraciones y con fluidez en el turno para usar la SCH. Para evitar malentendidos en la adquisición de sustancia o evitar peticiones de invitaciones con otros usuarios, se procura utilizar estrategias para ahuyentar a usuarios no convenientes, tales como no invitar nunca a drogas, no prestar dinero o no comprar sustancias a medias con personas que no son de confianza.

Yo flipo con la gente... vienen aquí a pedir dinero, droga o lo que sea. Yo nunca invito y ni me junto con nadie. Un día les invitas y luego a ti te hace falta y a lo mejor llevan y pasan de tu cara. Qué se busquen la vida y me dejen en paz. (Nota de campo, conversando con Paulus en la puerta de la $\mathrm{SCH}$ )

Yo prefiero consumir aquí [CAS Fòrum] porque es más acogedor. También vamos a la Mina [SCH] pero allí es un rollo. Son ocho plazas y mucha peña. Un día desapareció una bola [dosis] y el chico [profesional] de allí cerró el centro y dijo que de ahí no salía nadie hasta que no apareciera la bola... pero pasaron $30 \mathrm{mi-}$ nutos y nada. Seguro que ya se lo había metido cualquiera. No hay respeto por nadie. Aquí eso no te pasa... la sala es para una persona y tienes al enfermero a tu lado... no te puede pasar nada y no hay agobios. (Nota de campo, conversando con Miguel en la SCH).
En relación con las experiencias negativas de los efectos de las drogas, sobre todo en consumidores de cocaína, se procura preservar la tranquilidad dentro de los espacios de la $\mathrm{SCH}$, permanecer el tiempo mínimo y trasladarse a espacios más tranquilos, amplios y con menos estímulos que los del dispositivo, para experimentar más intensamente los efectos de esta sustancia.

\section{DISCUSIÓN}

A pesar del despliegue de programas e intervenciones de reducción de daños, los usuarios de drogas continúan manteniendo prácticas de riesgo con consecuencias negativas para la salud y padeciendo discriminación y estigmatización en el marco de las políticas prohibicionistas. Con este artículo se amplía el conocimiento sobre las prácticas y los discursos de los usuarios que consumen en vía pública, viviendas y $\mathrm{SCH}$, que revela elementos para promover acciones comunitarias de reducción de daños. Además de profundizar en las consecuencias negativas del consumo de drogas en diferentes "entornos de riesgo" y en las estrategias de autoatención que toman en cada caso, incorporar la noción de "placer" nos informa qué atrae a los usuarios a utilizar determinados programas de reducción de daños y cuáles pueden ser las innovaciones para responder mejor a las necesidades sociosanitarias. A continuación, se discute la gestión de los placeres y daños en los diferentes espacios de consumo $y$, en cada caso, se realizan propuestas para una intervención más efectiva.

En vía pública se detecta la presencia de personas con estilos de vida caracterizados por un consumo intenso, con graves problemas sociales y de salud ${ }^{(14,19)}$. Pese a considerarse un "entorno de riesgo", los usuarios permanecen en la búsqueda del bienestar mediante rituales cotidianos de consumo de drogas. Siguiendo a Duff(22), los placeres en la calle ocurren en situaciones en las que es factible preparar y consumir drogas en un ambiente que resulte atractivo. Para ello, los 
usuarios buscan lugares ocultos donde actuar libremente, sin ser fiscalizados y/o controlados, y sentir los efectos de las sustancias más intensamente. Este contexto moldea la experiencia de las personas consumidoras, las cuales llevan a cabo prácticas aceptadas entre pares que propician un consumo tranquilo y despreocupado, que no es posible en otros lugares ${ }^{(19)}$. Así, consumir en lugares sin ser recriminados y donde sea posible una experiencia sensorial intensa de los efectos de las drogas, es valorado positivamente por los usuarios. Estos aspectos son clave para optimizar el diseño y las normas de las $\mathrm{SCH} u$ otros programas de reducción de daños ${ }^{(14,41)}$.

Sin embargo, los espacios de consumo en vía pública representan los lugares con alto riesgo de contraer infecciones de transmisión sanguínea (VIH y hepatitis B y C) y sufrir sobredosis o reacciones adversas del consumo de drogas. Son lugares situados al margen a los que acuden los usuarios de drogas más visibles, que consumen por vía parenteral o pulmonar, prácticas consideradas estigmatizantes, que son el "chivo expiatorio" de aquellos que realizan consumos normalizados, y que tildan a los que consumen en la calle como problemáticos y difíciles de controlar ${ }^{(42)}$. En consecuencia, los usuarios consumen en lugares ocultos e insalubres, donde realizan prácticas de riesgo y sufren los daños a la salud descritos ${ }^{(19)}$. Por ello, es necesario reforzar y diversificar los programas de intercambio de jeringuillas para reducir la reutilización de material de consumo usado y promover una mayor difusión de mensajes preventivos en torno al consumo higiénico y seguro mediante talleres de prevención y atención de sobredosis, tal como apuntan publicaciones anteriores $\mathbf{s}^{(1,14,15,19,43)}$. Además, mientras no se produzcan cambios importantes en las políticas de drogas actuales, es necesario tomar acciones para reducir la vulnerabilidad entre las personas que usan drogas en vía pública. Un cambio fundamental sería una reforma política que dé paso hacia la despenalización, tal como se lleva a cabo en Portugal desde el año 2001, que puede favorecer un marco de intervención más pragmático y humanitario hacia los usuarios de drogas $^{(44)}$. De forma inmediata, se requiere la participación policial en el desarrollo de un entorno social propicio para la reducción de daños, tal como se recomiendan en otras publicaciones ${ }^{(17,45,46)}$.

Las viviendas son espacios considerados tranquilos e íntimos, en las que es posible obtener experiencias placenteras. Los usuarios relatan diversos placeres que varían según los estilos de vida ${ }^{(14)}$ y los diferentes tipos de viviendas que se disponen para consumir. Se detectan espacios domésticos de consumo, más o menos organizados, donde es posible el consumo higiénico de forma discreta, como son las viviendas familiares y en algunos pisos de venta en los que se permite consumir a clientes de confianza. A estos acuden personas con un consumo menos intenso y que presentan menor deterioro físico y cognitivo que los que acuden a las $\mathrm{SCH}$, lo realizan en vía pública o viviendas menos organizadas. No precisan supervisión en el consumo de drogas y procuran ocultar signos que los delate como consumidores ${ }^{(14,19)}$. Allí, experimentan los efectos deseados, con la capacidad de consumir tranquilos, estableciéndose buenas relaciones entre los consumidores. En pisos de venta de drogas regentados por vendedores, que son consumidores en situación de marginalidad, y en casas okupas poco organizadas, muchas veces con un aspecto de abandono, acceden usuarios con un consumo más intenso y con peores condiciones sociales y de salud que en los espacios anteriores. Sin embargo, las prácticas grotescas en estos ambientes no escapan de la lógica del placer ${ }^{(47)}$. En este tipo de viviendas tienen facilidad para adquirir drogas y es posible tener experiencias colectivas y obtener efectos más intensos de consumo, así como evitar los improperios en vía pública y las condiciones meteorológicas adversas. Además, se considera una alternativa a las $\mathrm{SCH}$, en las que se sienten vigilados y no se garantizan experiencias placenteras. Por otro lado, tampoco rechazan tomar medidas de reducción de daños, procurándose jeringuillas limpias y contenedores para desechar el material usado.

El mayor problema de salud del consumo de drogas en viviendas son las sobredosis por 
opioides. Como se evidencia en un informe sobre el consumo de drogas en la ciudad de Barcelona, el $73 \%$ de las muertes por sobredosis suceden en viviendas y el $75 \%$ son por consumo de heroína, detectándose una creciente presencia de más de dos sustancias ${ }^{(48)}$, si bien en los últimos años han disminuido las muertes por sobredosis de opioides, lo cual coincide con una mayor cobertura de talleres de prevención de sobredosis entre los usuarios ${ }^{(49)}$. Por lo tanto, para mantener y disminuir las sobredosis fatales es necesario continuar reforzando este tipo de talleres ${ }^{(43)}$. Además, para aumentar la cobertura, se recomiendan intervenciones basadas en la educación entre pares y la realización de talleres de consumo higiénico y seguro en casas okupas u otros espacios posibles, accediendo a través de usuarios captados desde los servicios de reducción de daños ${ }^{(50,51)}$, que tengan capacidad para actuar como agentes de salud y para aportar mayor credibilidad en los mensajes preventivos.

A las $\mathrm{SCH}$ acuden los usuarios de drogas con consumo intenso que presentan deterioro físico y cognitivo y que integran las estrategias de reducción de daños para la autoatención ${ }^{(2,14)}$. Los usuarios con mayor adhesión son aquellos que responden positivamente a los objetivos de estos dispositivos, que conceptualizan las $\mathrm{SCH}$ como espacios para un consumo higiénico y seguro, donde cubrir sus necesidades sociosanitarias y evitar los peligros sociales del consumo en vía pública y viviendas, como la violencia entre usuarios y la presión policial, tal como se expone en otros estudios basados en la experiencia de los usuarios ${ }^{(41,52,53)}$. Pero el vínculo no se produce bajo el instinto de abrazarse a los objetivos de las $\mathrm{SCH}$ y perseguir reducir los daños del consumo de drogas. Los usuarios acuden a aquellas $\mathrm{SCH}$ donde es posible obtener los efectos deseados del consumo y se propicia un ambiente tranquilo y de acompañamiento por parte de los profesionales. En dichas situaciones, los usuarios muestran no sentirse estigmatizados, creando un fuerte vínculo y compromiso con estos dispositivos, tal como se concluye en un estudio realizado en una $\mathrm{SCH}$ de Alemania ${ }^{(41)}$. Por lo tanto, en las $\mathrm{SCH}$ es posible consumir en condiciones higiénicas y seguras, y experimentar placeres que promueven a evitar entornos de riesgo, siendo estos argumentos importantes para continuar defendiendo estos dispositivos como espacios para reducir daños y mitigar la vulnerabilidad entre los consumidores de drogas.

Por el contrario, los usuarios relatan experiencias negativas en $\mathrm{SCH}$ donde no es posible disfrutar los efectos de las sustancias y tienen dificultades para sentirse reconocidos o libres, entre otros aspectos, que se tornan barreras de acceso a estos dispositivos. Se detecta que muchos usuarios prefieren consumir en vía pública u otros espacios para evitar el turno de espera, sobre todo en $\mathrm{SCH}$ masificadas, así como para no tener que asumir las normas de uso impuestas, que ya han sido detectadas en estudios en $\mathrm{SCH}$ de Canadá ${ }^{(45,52,53)}$. Para mejorar este aspecto es recomendable una mayor flexibilización en las normas de uso de las $\mathrm{SCH}$, de modo que se respete la toma de decisiones en la práctica de consumo y las idiosincrasias en la diversidad de usuarios ${ }^{(8,12,14,53)}$. Otra barrera detectada, es que las $\mathrm{SCH}$ no están suficientemente adaptadas para los usuarios de cocaína, dado que muchos refieren que son lugares molestos para disfrutar de la experiencia de consumo y, en consecuencia, prefieren consumir en vía pública u otros lugares. Este aspecto, indica la necesidad de diseñar $\mathrm{SCH}$ para el consumo de cocaína con mayor amplitud, funcionalidad y libre de elementos que alteren la percepción de los usuarios, tal como se recomienda en estudios anteriores ${ }^{(27,54,55)}$. A su vez, en la ciudad de Barcelona, solo se dispone de un espacio para el consumo de drogas por vía pulmonar, lo que supone una limitación para aquellos usuarios que adquieren drogas en barrios en los que no se dispone de $\mathrm{SCH}$ adaptadas a estos consumos, siendo recomendable la apertura de más espacios para el consumo por vía pulmonar, como ya ha sido recomendado en trabajos anteriores $^{(8,54,56)}$.

La investigación presentó varias limitaciones que deben ser consideradas. Una de ellas fue la imposibilidad de realizar observaciones en viviendas, en las que se podría haber 
recogido datos para profundizar en las experiencias de los usuarios de drogas en estos espacios. Esta limitación se superó parcialmente mediante la realización de entrevistas semiestructuradas en las que se preguntó sobre este aspecto. Sin embargo, se recomienda realizar investigaciones de carácter etnográfico sobre el consumo en viviendas para la reflexión del diseño de estrategias para un acercamiento de programas de reducción de daños a estos espacios. Además, se detectaron fallas en el diseño y las normas de las $\mathrm{SCH}$, que son barreras de acceso a estos dispositivos, que no fueron suficientemente analizadas y que requieren ser investigadas en trabajos posteriores. Pese a estos sesgos, esta investigación ha esclarecido elementos necesarios para la orientación en las políticas de reducción de riesgos y daños del consumo de drogas.

En conclusión, la comparación de las prácticas y discursos de los usuarios de drogas que consumen en vía pública, viviendas y $\mathrm{SCH}$, nos informa de los placeres y daños que experimentan y las estrategias de autoatención que emplean en los diferentes espacios de consumo. Centrarnos en los daños que sufren los usuarios y en cómo autorregulan las prácticas de consumo en los diferentes "entornos de riesgo", nos ha permitido conocer el impacto de los programas de reducción de daños y reflexionar acerca de cómo reforzar las estrategias de promoción de la salud. Pero, al incorporar la noción de "placer" hemos profundizado en las experiencias positivas del consumo en diferentes lugares y en qué atrae a utilizar determinados programas de reducción de daños. Estos aspectos deben tenerse en cuenta para diseñar y promover a nivel micro acciones comunitarias innovadoras de reducción de riesgos y daños más adaptadas a las necesidades sociosanitarias de los usuarios.

\section{REFERENCIAS BIBLIOGRÁFICAS}

1. Bosque-Prous M, Brugal MT. Intervenciones de reducción de daños en usuarios de drogas: situación actual y recomendaciones. Gaceta Sanitaria. 2016;30(Supl 1):104-110. doi: 10.1016/j.gaceta.2016.04.020.

2. Folch C, Lorente N, Majó X, Parés-Badell O, Roca X, Brugal T, Roux P, Carrieri P, Colom J, Casabona J, REDAN study group. Drug consumption rooms in Catalonia: A comprehensive evaluation of social, health and harm reduction benefits. International Journal of Drug Policy. 2018;62:24-29. doi: 10.1016/j.drugpo.2018.09.008.

3. Clua R, Tenza G, Mingueza J. Guia de bones pràctiques en els programes d'intercanvi de xeringues. Barcelona: Agencia de Salut Publica de Catalunya; 2015.

4. Dennis BB, Naji L, Bawor $M$, Bonner A, Varenbut $M$, Daiter J, Plater C, Pare G, Marsh DC, Worster A, Desai D, Samaan Z, Thabane L. The effectiveness of opioid substitution treatments for patients with opioid dependence: a systematic review and multiple treatment comparison protocol. Systematic Reviews. 2014;3:105. doi: 10.1186/2046-4053-3-105.

5. Potier C, Laprévote V, Dubois-Arber F, Cottencin O, Rolland B. Supervised injection services: what has been demonstrated?: A systematic literature review. Drug Alcohol Depend. 2014;145:48-68. doi: 10.1016/j.drugalcdep.2014.10.012.
6. Hedrich D, Kerr T, Dubois-Arbe F. Drug consumption facilities in Europe and beyond. En: Rhodes T, Hedrich D. (eds.) Harm reduction: Evidence, impacts and challenges. Luxembourg: European Monitoring Centre for Drugs and Drug Addiction; 2010. p. 305-331.

7. Noël L, Gagnon F, Bédard A, Dubé È. Avis sur la pertinence des services d'injection supervisée: analyse critique de la littérature. Québec: Institut national de santé publique du Québec; 2009.

8. Clua R. Salas de consumo higiénico en España (2000-2013). Salud Colectiva. 2015;11(2):261-282. doi 10.18294/sc.2015.688.

9. Bertrand B. Salles de shoot? Un regard engagé en faveur de l'expérimentation de structures d'accueil avec possibilité de consommer à moindre risque des drogues. Paris: Books on Demand; 2010.

10. Espelt A, Villalbí JR, Bosque-Prous $M$, Parés-Badell O, Mari-Dell'Olmo M, Brugal MT. The impact of harm reduction programs and police interventions on the number of syringes collected from public spaces: a time series analysis in Barcelona, 2004-2014. International Journal of Drug Policy. 2017;50:11-18. doi: 10.1016/j. drugpo.2017.07.033.

11. Vecino C, Villalbí JR, Guitart A, Espelt A, Bartroli $M$, Castellano Y, Brugal MT. Apertura de espacios de consumo higiénico y actuaciones policiales en zonas con fuerte tráfico de drogas: Evaluación mediante el re- 
cuento de las jeringas abandonadas en el espacio público. Adicciones. 2013;25(4):333-338. doi: 10.20882/ adicciones.35.

12. Anoro M, Ilundain E, Santisteban O. Barcelona's Safer InjectionFacility-EVA: A Harm Reduction Program Lacking Official Support. Journal of Drug Issues. 2003;33(3):689711. doi: 10.1177/002204260303300309.

13. Agència de Salut Pública de Catalunya. Informe anual 2017. Sistema d'informació sobre drogodependències a Catalunya. Barcelona: Generalitat de Catalunya; 2018.

14. Clua R. Estilos de vida de los usuarios de las salas de consumo higiénico de Barcelona. Health and Addictions, Salud y Drogas. 2018;18(2):79-89.

15. Folch C, Casabona J, Brugal MT, Majó X, Meroño M, Espelt A, González V. Perfil de los usuarios de drogas por vía parenteral que mantienen conductas de riesgo relacionadas con la inyección en Cataluña. Gaceta Sanitaria. 2012;26(1):37-44.

16. Haro JA. Cuidados profanos: una dimensión ambigua en la atención de la salud. En: Perdiguero E, Comelles J. (coord.). Medicina y cultura: estudios entre la antropología y la medicina. Barcelona: Bellaterra; 2000. p. 101-161.

17. Rhodes T. The 'risk' environment: A framework for understanding and reducing drug related harm. International Journal of Drug Policy. 2002;13:85-94. doi: 10.1016/S0955-3959(02)00007-5.

18. Rhodes T. Risk environments and drug harms: a social science for harm reduction approach. International Journal of Drug Policy. 2009;20(3):193-292. doi: 10.1016/j.drugpo.2008.10.003.

19. Llort Suárez A. «Dime dónde consumes y te diré...»: Antropología sobre el uso y abuso de cocaína. Perifèria, Revista de Recerca i Formació en Antropologia. 2018;23(1):56-83.

20. Sepúlveda M, Romaní O. Conceptualización y políticas de la gestión del riesgo. En: Martínez Oró DP, Pallarés J, (eds.). De riesgos y placeres: manual para entender las drogas. Lleida: Milenio; 2013. p. 89-102.

21. Duff C. Towards a theory of drug use contexts: Space, embodiment and practice. Addiction Research and Theory. 2007;15(5):503-519. doi: 10.1080/16066350601165448.

22. Duff $C$. The pleasure in context. International Journal of Drug Policy. 2008;19(5):384-392. doi: 10.1016/j. drugpo.2007.07.003.

23. Martínez Oró DP, Pallarés J. Riesgos, daños y placeres. En: Martínez Oró DP, Pallarés J, (eds.). De riesgos y placeres: manual para entender las drogas. Lleida: Milenio; 2013. p. 23-36.

24. Dilkes-Frayne E. Tracing the "event" of drug use: "Context" and the coproduction of a night out on MDMA. Contemporary Drug Problems. 2014;41(3):445479. doi: 10.1177/009145091404100308.
25. Martínez Oró DP, Romaní O. Els danys de les polítiques prohibicionistes en I'àmbit de les drogues. Quaderns-E. 2016;21(1):33-49.

26. Clua R. ¡Apúntame a la sala!: Epidemiología sociocultural del consumo de drogas y evaluación de las políticas de reducción de daños en las salas de consumo higiénico de Barcelona. [Tesis Doctoral]. Departament d'Antropologia Social i Cultural de la UAB; 2016.

27. Clua R. Atención de enfermería del síndrome posconsumo de cocaína en usuarios de las salas de consumo higiénico de Barcelona. IV Congreso Virtual Internacional de Enfermería en Salud Mental [Internet]. 2018;4 [citado 10 may 2019]. Disponible en: http:// psiqu.com/1-8944

28. Bourgois P, Schonberg J. Righteous Dopefiend. Berkeley: University of California Press; 2009.

29. Carlson RG, Singer M, Stephens RC, Sterk CE. Reflections on 40 years of ethnographic drug abuse research: Implications for the future. Journal of Drug Issues. 2009;39(1):57-70. doi: 10.1177/002204260903900106.

30. Singer M. Anthropology and addiction: An historical review. Addiction. 2012;107(10):1747-1755. doi: 10.1111/j.1360-0443.2012.03879.x.

31. Romaní O. La epidemiología sociocultural en el campo de las drogas: contextos, sujetos y sustancias. En: Haro JA, (coord.). El planteamiento de una epidemiología sociocultural. Un diálogo en torno a su sentido, métodos y alcances. Buenos Aires: Lugar; 2010. p. 89113.

32. Agència de Salut Pública de Catalunya. Informe anual 2015. Sistema d'informació sobre drogodependències a Catalunya. Barcelona: Generalitat de Catalunya; 2016.

33. Martínez-Salgado C. El muestreo en investigación cualitativa: principios básicos y algunas controversias. Ciência \& Saúde Coletiva. 2012;17(3):613-619. doi: 10.1590/S1413-81232012000300006.

34. Shaghaghi A, Bhopal RS, Sheikh A. Approaches to recruiting "Hard-To-Reach" populations into research: A review of the literature. Health Promotion Perspectives. 2011;1(2):86-94. doi: 10.5681/hpp.2011.009.

35. Hammersley M, Atkinson P. Ethnography: principles in practice. Third edition. London: Routledge; 2007.

36. Emerson RM, Fretz R, Shaw LL. Writing ethnographic fieldnotes. Chicago: University of Chicago Press; 2011.

37. DiCicco-Bloom B, Crabtree BF. The qualitative research interview. Medical Education. 2006;40(4):314321. doi: 10.1111/j.1365-2929.2006.02418.x.

38. Jamshed S. Qualitative research method-interviewing and observation. Journal of Basic and Clinical Pharmacy. 2014;5(4):87-88. doi: 10.4103/0976-0105.141942.

39. Bengtsson M. How to plan and perform a qualitative study using content analysis. NursingPlus Open. 2016:2:8-14. doi: 10.1016/j.npls.2016.01.001. 
40. Carter N, Bryant-Lukosius D, DiCenso A, Blythe J, Neville AJ. The use of triangulation in qualitative research. Oncology Nursing Forum. 2014;41(5):545-547. doi: 10.1188/14.ONF.545-547.

41. Duncan T, Duff C, Sebar B, Lee J. 'Enjoying the kick' Locating pleasure within the drug consumption room. International Journal of Drug Policy. 2017;49:92-101. doi: 10.1016/j.drugpo.2017.07.005.

42. Martínez Oró DP. Sin pasarse de la raya. La normalización de los consumos de drogas. Barcelona: Bellaterra; 2015 .

43. Sarasa-Renedo A, Espelt A, Folch C, Vecino C, Majó X, Castellano Y, Casabona J, Brugal MT, Redan Study Group. Overdose prevention in injecting opioid users: the role of substance abuse treatment and training programs. Gaceta Sanitaria. 2014;28(2):146-54. doi: 10.1016/j.gaceta.2013.10.012.

44. Domoslawski A. Drug policy in Portugal: The benefits of decriminalizing drug use. Warsaw: Open Society Foundations; 2011.

45. Bardwell G, Strike C, Altenberg J, Barnaby L, Kerr $\mathrm{T}$. Implementation contexts and the impact of policing on access to supervised consumption services in Toronto, Canada: a qualitative comparative analysis. Harm Reduct J. 2019;16(1):30.

46. Shaw A, Lazarus L, Pantalone T, LeBlanc S, Lin D, Stanley D, Chepesiuk C, Patel S, Tyndall M. Risk environments facing potential users of a supervised injection site in Ottawa, Canada. Harm Reduction Journal. $2015 ; 12: 49$

47. Moore D. Erasing pleasure from public discourse on illicit drugs: On the creation and reproduction of an absence. International Journal of Drug Policy. 2008;19(5):353-358. doi: 10.1016/j.drugpo.2007.07.004

48. Brugal MT, Guitart AM, Espelt A, (reds.). Pla d'Acció sobre Drogues de Barcelona 2013-16. Barcelona: Agència de Salut Pública de Barcelona; 2013.
49. Espelt A, Major X, Parés-Badell O, Carvajal S, Gasulla $\mathrm{L}$, Bosque-Prous $\mathrm{M}$, Brugal, $\mathrm{M}$. T. Implementation of Systematic Programs of Overdose Training at Drug Treatment and Prevention Centres in Catalonia, 20082013. En: Dichtl A, Stöver H (eds.). Naloxon - Überlebenshilfe im Drogennotfall. Frankfurt: Fachhochschulverlag; 2015. p. 83-95.

50. Holloway K, Hills R, May T. Fatal and non-fatal overdose among opiate users in South Wales: A qualitative study of peer responses. The International Journal on Drug Policy. 2018;56:56-63. doi: 10.1016/j.drugpo.2018.03.007.

51. Latkin CA, Gicquelais RE, Clyde C, Dayton L, DaveyRothwell M, German D, Falade-Nwulia S, Saleem H, Fingerhood M, Tobin K. Stigma and drug use settings as correlates of self-reported, non-fatal overdose among people who use drugs in Baltimore, Maryland. International Journal of Drug Policy. 2019;68:86-92. doi: 10.1016/j.drugpo.2019.03.012.

52. Jozaghi E, Andresen MM. Should North America's first and only supervised injection facility (InSite) be expanded in British Columbia, Canada? Harm Reduction Journal. 2013;10:1. doi: 10.1186/1477-7517-10-1.

53. Small W, Moore D, Shoveller J, Wood E, Kerr T. Perceptions of risk and safety within injection settings: Injection drug users' reasons for attending a supervised injecting facility in Vancouver, Canada. Health, Risk \& Society. 2012;14(4):307-24. doi: 10.1080/13698575.2012.680950.

54. Delàs J, Priore AG, Pigem A, Aguas M. Síndrome posconsumo de cocaína inyectada a partir de las observaciones en una sala de consumo supervisada. Medicina Clínica. 2008;130(1):35. doi: 10.1157/13114543.

55. Roncero C, Martínez-Luna N, Daigre C, GrauLópez L, Gonzalvo B, Pérez-Pazos J, Casas M. Psychotic symptoms of cocaine self-injectors in a harm reduction program. Substance Abuse. 2013;34(2):118-121. doi: $10.1080 / 08897077.2012 .691446$

56. Delàs J, Adán E, Díaz $O$, Aguas $M$, Pons $M$, Fuertes R. Smoked cocaine in socially-depressed areas. Harm Reduction Journal. 2010;7:27. doi: 10.1186/1477-7517-7-27.

FORMA DE CITAR

Clua-García R. La gestión de placeres y daños: Estudio etnográfico sobre el consumo de drogas en vía pública, viviendas y salas de consumo higiénico. Salud Colectiva. 2020;16:e2481. doi: 10.18294/sc.2020.2481.

Recibido: 20 ago 2019 | Versión final: 19 may 2020 | Aprobado: 16 jun 2020 | Publicado en línea: 21 jul 2020

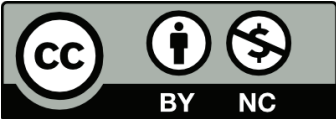

Esta obra está bajo una licencia de Creative Commons Reconocimiento-NoComercial 4.0 nternacional. Reconocimiento - Permite copiar, distribuir y comunicar públicamente la obra. A cambio, se debe reconocer y citar al autor original. No Comercial - Esta obra no puede ser utilizada con finalidades comerciales, a menos que se obtenga el permiso. 